\title{
PO2-7-3
}

Poster session

\section{Comparative equivalence of Metformin brands available in Sokoto, Northwestern Nigeria}

\section{Abdulhakeem Adio Awesu ${ }^{1}$, Bello Oricha Shaibu ${ }^{1}$, Chika Aminu ${ }^{1}$, Acho Mohammed ${ }^{2}$, Akinleye Olushola Moshood ${ }^{3}$}

${ }^{I}$ Department of Pharmacology and Therapeutics, College of Health Sciences, Usmanu Danfodiyo University, Sokoto,
Nigeria, ${ }^{2}$ Department of Pharmaceutical Microbiology, Faculty of Pharmaceutical Sciences, Usmanu Danfodiyo
University., Nigeria, ${ }^{3}$ Department of Pharmaceutical Chemistry, Faculty of Pharmacy, University of Lagos, Nigeria

Comparative Equivalence Of Metformin Brands Available In Sokoto,

Northwestern Nigeria.

A.A. Awesu, S.O. Bello, A. Chika, M. Achor, M.O. Akinleye

Introduction

High levels of substandard or fake generics are often touted as the rule in Africa.This may not be true for all drugs. The aim of this study was to establish if Pharmaceutical and/or bioequivalence exist among brands of metformin marketed in Sokoto Northwestern Nigeria.

Methods

Eight available brands of Metformin were compared to innovator Glucophage. The drugs were subjected to invitro pharmaceutical equivalence studies. Also, a randomised, single dose, two-period, crossover study in 10 healthy Sokoto red goats was designed for the brand with the best pharmaceutical equivalence (for logistic reasons).A two-weeks washout period was maintained. A single dose of $500 \mathrm{mg}$ of each brand was administered, plasma samples up to 24 hours $(0$, 0.5,1,2,4,8,12 and 24) were taken.Plasma drug concentrations were determined using a validated High Pressure Lipid Chromatography, UV detection method.

\section{Results}

All the brands met the British Pharmacopoeia (BP) specifications for uniformity of weight, disintegration and dissolution test, however for the friability test, one of the eight brands failed.All the brands had values within the range for assay in BP. All the brands equally passed the disintegration, weight uniformity and dissolution studies. However, only four brands demonstrated equivalence at $\mathrm{pH} 6.8$ based on F2 similarity factor. Analysis of individual pharmacokinetic parameters revealed no significant difference between Glucophage and Glumin at 90\% confidence interval(CI). The ratios of average Cmax, AUC0-t and AUC0- of Metformin samples (test against reference) for the logarithm transformed data were 1.01,1 and 1 respectively. The corresponding 90\% CI were $0.9512-1.0781,0.978-1.022$ and $0.9667-1.0251$ which were within the acceptable range of $0.80-1.25$.

Conclusions

This study demonstrated pharmaceutical equivalence between the Metformin formulations and therapeutic equivalence between Glucophage and Glumin.

References

1. Jung SH et al. (2014). Iran J Pharm Res. 13:365-71.

2. Idkaidek NM (2017). Saudi Pharm J. 25:671-675

3. Batolar LS et al. (2012). Arzneimittelforschung. 62:22-6.

4. Homsek I et al. (2010). Arzneimittelforschung. 60:553-9

5. Li J et al.(2007). Eur J Drug Metab Pharmacokinet. 32:21-8. 